\title{
Monoarthritis revealing Infliximab-induced systemic lupus erythematosus in Crohn's disease
}

\begin{abstract}
Introduction: Infliximab is increasingly used for the treatment of severe and resistant forms of Crohn's disease (CD). It is characterized by its efficiency and good tolerance. Induced systemic lupus erythematosus (SLE) remains an exceptional but potentially serious complication of this treatment and its diagnosis represents a real challenge for clinicians.

We are reporting an original observation of infliximab-induced SLE in CD whose revealing clinical presentation was an isolated monoarthritis.

Case report: A 28-year-old woman, followed for severe and resistant to first-line treatment CD since the age of 20 . Treatment with infliximab was thus initiated with a satisfactory effect. During her admission for the fifth infusion of infliximab, she reported inflammatory arthralgia in the small joints of her hands. Somatic examination noted an isolated arthritis of the proximal interphalangeal joint of the right fifth finger. Biology noted leukopenia at 2000/ $\mathrm{mm}^{3}$, neutropenia at $1250 / \mathrm{mm}^{3}$, lymphopenia at $634 / \mathrm{mm}^{3}$, and erythrocyte sedimentation rate at $68 \mathrm{~mm} / \mathrm{H1}$. Anti-nuclear antibodies were positive at $1 / 640$ and anti-double stranded DNA antibodies were positive at $1 / 160$. Thus, the diagnosis of infliximab-induced SLE was retained. Infliximab was discontinued, and the outcome was favorable under systemic corticosteroid therapy associated with Hydroxychloroquine and Mesalazine.
\end{abstract}

Conclusion: As rare as it is, this complication of biotherapy deserves to be known by clinicians and the diagnosis deserves to be mentioned in front of any monoarthritis occurring in CD patient under anti-TNF $\alpha$ therapy.

Keywords: monoarthritis, infliximab, systemic lupus erythematosus, Crohn disease, drug-induced lupus
Volume II Issue 5 - 2019

\author{
Salem Bouomrani, ${ }^{1,2}$ Fatma Rekik, ${ }^{1,2}$ Rim \\ Mesfar, ${ }^{1,2}$ Ali Naffati, ${ }^{1,2}$ Warda Mahdhaoui, ${ }^{1,2}$ \\ Amin Hammami, ${ }^{1,2}$ DhiaEddine Amri, ${ }^{1,2}$ \\ 'Department of Internal medicine, Military Hospital of Gabes, \\ Tunisia \\ ${ }^{2}$ Sfax Faculty of Medicine, University of Sfax, Tunisia
}

Correspondence: Salem Bouomrani, Department of Internal medicine, Military Hospital of Gabes, Gabes 6000,Tunisia,Tel +0021698977555, Email salembouomrani@yahoo.fr

Received: September 14, 2019| Published: October 01, 2019
Abbreviations: CD, Crohn's disease; SLE, systemic lupus erythematosus; UC, ulcerative colitis; ANA, anti-nuclear antibodies; anti-DNA, DNA antibodies

\section{Introduction}

Infliximab, a chimeric monoclonal antibody, is an anti-tumor necrosis factor alpha (anti-TNF $\alpha$ ) that is increasingly used for the treatment of severe and resistant forms of inflammatory bowel disease (Crohn's disease (CD) and ulcerative colitis (UC)). ${ }^{1,2}$ It is characterized by its efficiency and good tolerance. ${ }^{2}$ Induced systemic lupus erythematosus (SLE) remains an exceptional but potentially serious complication of this biological treatment.,

The diagnosis of these cases of infliximab-induced SLE in patient treated for $\mathrm{CD}$ represents a real challenge for clinicians for several reasons:

a. Similar clinical signs and symptoms, the possibility of gastrointestinal involvement during lupus, the possibility of lupus-like extra-digestive manifestations during $\mathrm{CD}$, and the overlapping diagnosis criteria for SLE and CD,3,4

b. The possibility of association of these two diseases in the same patient. ${ }^{3,4}$ The frequency of concomitant diagnosis of inflammatory bowel diseases and SLE is estimated at $0.4 \%$ and seems to be more frequent for $\mathrm{UC}$ than $\mathrm{CD},{ }^{4}$

c. Clinical presentations of induced lupus are often incomplete and nonspecific. ${ }^{5,6}$
We are reporting an original observation of infliximab-induced SLE in a patient treated for CD, whose revealing clinical presentation was a monoarthritis.

\section{Case report}

A 28-year-old Tunisian woman, followed for CD since the age of 20 , with severe and resistant to first-line treatment disease. His disease was complicated by colonic involvement with corticosteroid-resistant colitis requiring subtotal colectomy, and fistulising ano-perineal involvement, which was not improved by tri-therapy involving antibiotics, systemic glucocorticoids, and azathioprine.

Treatment with infliximab at a dose of $5 \mathrm{mg} / \mathrm{kg}$ by infusion was thus initiated with a satisfactory effect. During her admission for the fifth infusion of infliximab, the patient reported inflammatory arthralgia in the small joints of her hands. Somatic examination noted arthritis of the proximal interphalangeal joint of the right fifth finger (Figure $1 \&$ 2). The other joints were free. No local trauma of the affected finger or periarticular skin lesions were noted. The patient was afebrile, and had no digestive symptoms or extra-digestive manifestations of her CD. The X-ray of the hands did not show any fracture, joint destruction, or deformity.

Routine biology noted leukopenia at $2000 / \mathrm{mm}^{3}$, neutropenia at $1250 / \mathrm{mm}^{3}$, lymphopenia at $634 / \mathrm{mm}^{3}$, and erythrocyte sedimentation rate at $68 \mathrm{~mm} / \mathrm{H} 1$. The remainder of the bioassays was within normal limits: red blood cells, hemoglobin, platelets, C-reactive protein, serum calcium, glucose, ionogram, transaminases, muscle enzymes, lipid 
parameters, uric acid, creatinine, and serum protein electrophoresis Rheumatoid factor and anti-cyclic citrullinated peptide antibodies were negative. Anti-nuclear antibodies (ANA) were positive at 1/640 and anti-double stranded DNA antibodies (anti-DNA) were positive at $1 / 160$. Serum complement fractions $(\mathrm{C} 3, \mathrm{C} 4, \mathrm{C} 2$, and $\mathrm{CH} 50)$ were without significant abnormalities.

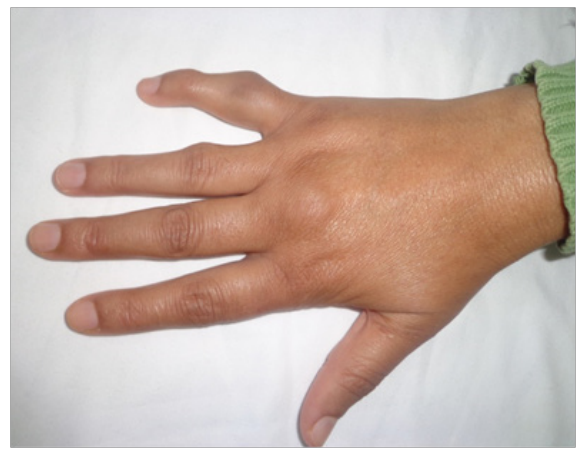

Figure I Tumefaction of the proximal interphalangeal joint of the right fifth finger.

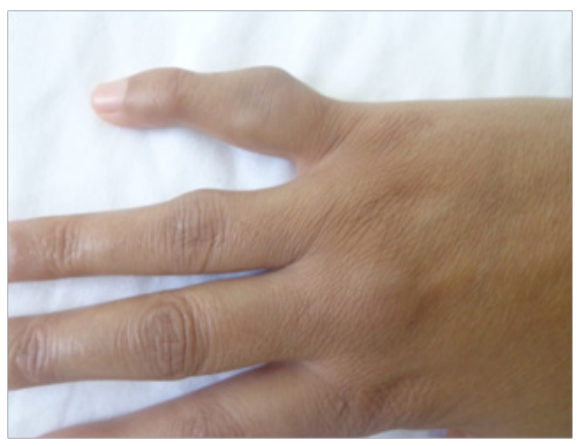

Figure 2 Monoarthritis of the proximal interphalangeal joint of the right fifth finger.

Thus, the diagnosis of infliximab-induced lupus was retained: non-erosive arthritis, leukopenia, neutropenia, lymphopenia, positive ANA, and positive anti-DNA, as well as the notion of a negative immunological assessment one year before the introduction of infliximab. Anti-histone antibodies were positive confirming the diagnosis. Infliximab therapy was discontinued, and systemic corticosteroid therapy at a dose of $1 \mathrm{mg} / \mathrm{kg} /$ day associated with Hydroxychloroquine and Mesalazine was started with favorable outcome.

\section{Discussion}

Drug-induced autoimmune disorders have been reported as possible adverse reactions of biotherapy, but true induced-autoimmune diseases remain rare. $^{1}$ In $\mathrm{CD}$ and infliximab-treated patients, production of anti-nuclear and anti-DNA auto-antibodies may be noted up to $53 \%$ and $35 \%$ respectively. ${ }^{4}$ These auto-antibodies do not usually have pathological significance, and the absence of associated clinical signs justifies that regular monitoring in these patients is not recommended. ${ }^{1,4}$

Rarely, some CD and infliximab-treated patients may develop minor forms of lupus disease such as cutaneous lupus or lupus-like syndrome. ${ }^{4,5}$ The induction of true SLE cases with infliximab remains exceptional. ${ }^{4,6,7}$ Indeed, in the large series of the Mayo Clinic of 500 patients with $\mathrm{CD}$ treated by infliximab, only three $(0.6 \%)$ developed induced SLE. ${ }^{2}$
Infliximab-induced SLE has also been reported in other inflammatory bowel diseases such as UC, and with other antiTNF $\alpha$ therapies such as Adalimumab and Etanercept. ${ }^{1,4}$ The most suggested pathophysiological mechanism for explaining these cases of infliximab-induced SLE is the likely effects of blocking TNF $\alpha$ on anti-DNA auto-antibodies production. ${ }^{4}$ Classically, the evolution of induced SLE is rapidly favorable after the withdrawal of infliximab with rapid disappearance of clinical signs and negation of serological and immunohistochemical markers of lupus.,5-7 Exceptionally prolonged forms of infliximab-induced lupus disease have been reported. ${ }^{8}$

Therapeutic eventualities in these cases are either switching to another anti-TNF alpha or systemic corticosteroid therapy with azathioprine or Mesalazine. ${ }^{5-8}$ Our observation is distinguished by a monosymptomatic clinical presentation of infliximab-induced SLE in CD. To our knowledge, isolated monoarthritis of the interphalangeal joint has not been reported previously as a warning sign of induced lupus in patients with infliximab-treated DC.

\section{Conclusion}

Infliximab-induced SLE remains an exceptional but potentially serious complication in patients with $\mathrm{CD}$. Monosymptomatic and incomplete presentations represent a real diagnostic challenge. As rare as it is, this complication of biotherapy deserves to be known by clinicians and the diagnosis deserves to be mentioned in front of any monoarthritis occurring in $\mathrm{CD}$ patient under anti-TNF $\alpha$ therapy.

\section{Acknowledgments}

None.

\section{Conflicts of interest}

The authors declare there is no conflict of interest.

\section{References}

1. Nishikawa J, Miyazaki T, Suzuki N, et al. Infliximab-induced lupus erythematosus in a case of ulcerative colitis. Nihon Shokakibyo Gakkai Zasshi. 2011;108(9):1535-1539.

2. Colombel JF, Loftus EV Jr, Tremaine WJ, et al. The safety profile of infliximab in patients with Crohn's disease: the Mayo clinic experience in 500 patients. Gastroenterology. 2004;126(1):19-31.

3. João-Magalhães M, Lago P, Pedroto I. Crohn's disease and systemic lupus erythematosus: A rare and challenging association. Rev Esp Enferm Dig. 2015;107(6):394-395.

4. Katsanos KH, Voulgari PV, Tsianos EV. Inflammatory bowel disease and lupus: a systematic review of the literature. J Crohns Colitis. 2012;6(7):735-742.

5. Cemil BC, Atas H, Canpolat F, et al. Infliximab-induced discoid lupus erythematosus. Lupus. 2013;22(5):515-518.

6. Naseer M, Kulairi Z, Kam M. Cardiac tamponade as a presenting manifestation of infliximab-induced lupus in patient treated for Crohn's disease. ACG Case Rep J. 2017;4:e1.

7. Choi SJ, Kim S, Kim HY, et al. Infliximab-induced lupus in Crohn's disease. Korean J Med. 2019;94(3):303-307.

8. Sarzi-Puttini P, Ardizzone S, Manzionna G, et al. Infliximab-induced lupus in Crohn's disease: a case report. Dig Liver Dis. 2003;35(11):814 817. 